\title{
Quasinormal modes and quantization of area/entropy for noncommutative BTZ black hole
}

\author{
Lu Huang, Juhua Chen ${ }^{\text {a }}$, Yongjiu Wang
}

Department of Physics, Key Laboratory of Low Dimensional Quantum Structures and Quantum Control of Ministry of Education, and Synergetic Innovation Center for Quantum Effects and Applications, Hunan Normal University, Changsha 410081, Hunan, People’s Republic of China

Received: 30 January 2018 / Accepted: 4 April 2018 / Published online: 17 April 2018

(C) The Author(s) 2018

\begin{abstract}
We investigate the quasinormal modes and area/ entropy spectrum for the noncommutative BTZ black hole. The exact expressions for QNM frequencies are presented by expanding the noncommutative parameter in horizon radius. We find that the noncommutativity does not affect conformal weights $\left(h_{L}, h_{R}\right)$, but it influences the thermal equilibrium. The intuitive expressions of the area/entropy spectrum are calculated in terms of Bohr-Sommerfeld quantization, and our results show that the noncommutativity leads to a nonuniform area/entropy spectrum. We also find that the coupling constant $\xi$, which is the coupling between the scalar and the gravitational fields, shifts the QNM frequencies but not influences the structure of area/entorpy spectrum.
\end{abstract}

\section{Introduction}

In classical gravitational theory, perturbations of black holes or branes naturally lead to quasinormal modes (QNM) which are eigenmodes of dissipative systems [1]. It's well known that QNM is a significant tool to study $A d S / C F T$ duality [2-6]. In Refs. [7-9], the QNM frequencies determine the decay of the gravitational perturbations in the bulk and relaxation time of the two-point function of the thermal $C F T$ at the boundary of BTZ black holes. The quasinormal modes provide a correspondence between the perturbation of the gravity in the bulk to that of a boundary CFT in BTZ spacetime. While this result provides a proposal for the AdS/CFT duality, it would be significant to test its validity at the Planck scale, which is a natural regime for holography and quantum gravity. Noncommutative geometry is one of the candidates of the quantum gravity at the Planck scale [10]. In particular, string theory, loop gravity [11] and noncommutative geometry [10] suggest that the space-time might have some discrete structures at the Planck scale, thus the validity of $A d S / C F T$

\footnotetext{
a e-mail: jhchen@hunnu.edu.cn
}

duality becomes very important. It is widely believed that the noncommutative space-time can be described by involving the quantum theory and general relativity together [12]. For instance, the noncommutative BTZ background can be described by $\kappa$-Minkowski algebra [13-15]. Therefore, the investigations on $\kappa$-Minkowski QNM in BTZ background turn out to be extremely important, which should be based on $\kappa$-Minkowski framework [16,17].

In Ref. [18], the unitarity of scalar noncommutative field theories has been checked at the one loop level. They found that noncommutative field theories with only space noncommutativity (that is $\theta^{0 i}=0$ ) are perturbatively unitary. And space-time noncommutative field theories (that is $\theta^{0 i} \neq 0$ ) do not have a unitary S-matrix and do not satisfy the cutting rules for Feynman diagrams. What's more, there is a subtle point that two limits don't necessarily commute, one is the process of putting the deformation parameter to zero, and the other is to perform the integral in the calculation of various Feynman diagrams (where also the UV/IR mixing enters) $[19,20]$. An example of a finite theory for finite noncommutative parameter was also considered in Refs. [21,22]. In other words, space noncommutative field theories describe a low energy limit of string theory in a background magnetic field. String theory can not be appropriately described by spacetime noncommutative field theories (see [18]). Usually most of the analyses from string theory to noncommutative field theory are done with the assumption that the background flux is constant. However, when the background flux coming from the string theory is not constant, Aschieri et al. [23], with developing the metric aspects of non-associative differential geometry tailored to the parabolic phase space model of constant locally non-geometric closed string vacua, obtained explicit expressions for the torsion, curvature, Ricci tensor and Levi-Civita connection in non-associative Riemannian geometry on phase space, constructed R-flux corrections to the Ricci tensor on space-time, and commented on the poten- 
tial implications of these structures in non-geometric string theory. By studying the noncommutative scalar and fermionic quasinormal modes in commutative BTZ black hole, Gupta et al. [24,25] exploited a duality between a non-rotating and rotating BTZ black hole, the spin being proportional to the noncommutative deformation parameter. Therefore, their works showed how the Plank scale physics might influence the QNM frequency by setting the correct commutative limit and noncommutative corrections up to the first order in the deformation parameter. For example, they showed that the horizon temperatures in the dual $C F T$ are modified due to noncommutative contributions through the $A d S / C F T$ correspondence. Furthermore, they demonstrated the equivalence between the quasinormal and non-quasinormal modes for the noncommutative fermionic probes, which provides further evidence of holography in the noncommutative setting.

In quantum gravity, the black hole area/entropy quantization is always connected to QNM frequency, which is originally predicted by Bekenstein who assumed the event horizon as an adiabatic invariant [26]. An important character of loop quantum gravity is that the spectrum of geometrical quantities is not continued but discrete [27-29]. According to the correspondence principle, Hod proposed that the real part of the highly damped QNM might be related to the fundamental quanta of mass and angular momentum [30]. Particularly, the area/entropy spectrum has been deeply studied in the nonrotating BTZ background [31]. They have taken advantage of Boher-Sommerfeld quantization for an adiabatic invariant and given an equally spaced mass spectrum, which is different from 3+1-dimension black hole. Bekenstein [26] argued that the QNM frequency $\omega_{(E)}$, in high damping limit, should take the form of $\omega_{(E)}=\sqrt{\left|\omega_{R}\right|^{2}+\left|\omega_{I}\right|^{2}}$. Therefore, the QNM frequency $\omega_{(E)}$ approximately equaled $\omega_{R}$ when $\omega_{I} \rightarrow 0$. And the frequency would become $\omega_{E}=\omega_{I}$ in the case of $n \rightarrow \infty$.

In this paper, motivated by that the noncommutative field causes a corrections to the QNM of BTZ black hole and slightly affects the area/entropy spectrums of BTZ black hole [24], we mainly focus our attentions on noncommutative BTZ black hole with the nonminimally coupling scalar field and massless Dirac field. We plan to calculate the exact expressions for QNM frequencies in different cases and investigate area/entropy spectrum with the existence of noncommutativity in terms of Bohr-Sommerfeld quantization. The paper is organized as follows: in Sec. 2, we briefly introduce the noncommutative BTZ black hole. In Sec. 3, we analytically calculate the QNM of this black hole with nonminimally coupling scalar field. In Sec. 4, we present the exact expressions of QNM frequencies of this black hole with massless Dirac field. In Sec. 5, the quantitative agreement in the noncommutative BTZ background is discussed. In Sec. 6, the area/entropy spectrum of this black hole is investigated in terms of the QNM presented. At last, a brief conclusion is given.

\section{Noncommutative BTZ black hole}

At first we give a brief review on the Refs. [18,32] as follows. Noncommutative field theories are constructed from commutative field by replacing in the lagrangian the usual multiplication of fields with the $\star$-product of fields, which is defined in terms of a real antisymmetric matrix $\theta^{\mu \nu}$ that parameterizes the noncommutativity of Minkowski space-time [18]. The commutation relation for noncommutative space-time (we adopt the formula in Ref. [32]) takes the form of

$$
\left[\hat{x}^{\mu}, \hat{x}^{\nu}\right]=i \theta^{\mu \nu},
$$

where $\mu, v=0, \ldots, D-1$. The $\star$-product of two functions on noncommutative space-time is given by

$\left.(f \star g)(x) \equiv e^{\frac{i}{2} \theta^{\mu \nu} \frac{\partial}{\partial x^{\mu}} \frac{\partial}{\partial y^{\nu}}} f(x) g(x)\right|_{x=y}$.

Seiberg [33] showed that there exists an equivalence between ordinary gauge fields and noncommutative gauge fields, Seiberg-Witten map [33] is a useful tool to study various properties of noncommutative gauge theories. However, one can not use Seiberg-Witten map to noncommutative gravity directly. One way of evading this is to regard the Einstein's gravity as the Poincaré gauge theory and apply the SeibergWitten map for its noncommutative extension [34] or take the twisted Poincaré algebra approach in Refs. [35-37] based on Refs. $[38,39]$. Only in the three dimensional case, one can directly deal with the gravity by using Seiberg-Witten map because of the equivalence between the three dimensional gravity theory and the Chern-Simons theory, which has been mentioned in Refs. [40,41]. The noncommutative extension of this equivalence was investigated in Refs. [42,43]. Based on the three dimensional equivalence between gravity and Chern-Simons theory, the authors in Ref. [32] have studied the noncommutative BTZ black hole solutions in polar coordinates $(\hat{r}, \hat{\phi}, t)$ with the following commutation relation

$[\hat{r}, \hat{\phi}]=i \theta \hat{r}^{-1}$.

The relationship between the polar coordinates and the rectangular coordinates in noncommutative space has been explained. They showed that the commutation relations Eq. (3) are equivalent to the canonical commutation relation of the rectangular coordinates

$[\hat{x}, \hat{y}]=i \theta$.

Reference [43] showed that, in the commutative limit, the $(2+1)$ dimensional $U(1,1) \times U(1,1)$ noncommutative 
Chern-Simons theory equals to the three dimensional Einstein's gravity plus two decoupled $U(1)$ theory. The extension of the commutative $S U(1,1) \times S U(1,1)$ Chern-Simons theory has to contain $U(1)$ elements. Thus, the noncommutative extension of $S U(1,1) \times S U(1,1)$ Chern-Simons theory has to be $U(1,1) \times U(1,1)$ noncommutative Chern-Simons theory. Therefore, one can regard the $U(1,1) \times U(1,1)$ noncommutative Chern-Simons theory as a noncommutative extension of the three dimensional Einstein's gravity. And the solution of the noncommutative Chern-Simons theory can be obtained from its commutative counter by using the Seiberg-Witten map [33]. The metric of the noncommutative BTZ black hole is given by Ref. [32]

$$
\begin{aligned}
d s^{2}=- & f^{2} d t^{2}+N^{-2} d r^{2}+2 r^{2} N^{\phi} d t d \phi+\left(r^{2}+\frac{\theta B}{2}\right) d \phi^{2} \\
& +\mathcal{O}\left(\theta^{2}\right),
\end{aligned}
$$

where

$$
\begin{aligned}
N^{\phi}= & -\frac{r_{+} r_{-}}{l r^{2}}, \\
f^{2}= & \frac{r^{2}-r_{+}^{2}-r_{-}^{2}}{l^{2}}-\frac{\theta B}{2 l^{2}}, \\
N^{2}= & \frac{1}{l^{2} r^{2}}\left[\left(r^{2}-r_{+}^{2}\right)\left(r^{2}-r_{-}^{2}\right)\right. \\
& \left.-\frac{\theta B}{2}\left(2 r^{2}-r_{+}^{2}-r_{-}^{2}\right)\right],
\end{aligned}
$$

where $B$ is the magnitude of the magnetic field, $\theta$ is the noncommutative parameter. The details of the background magnetic field can be found in Refs. [33,44,45]. $r_{+}$and $r_{-}$ are the radius of outer and inner horizons

$r_{ \pm}^{2}=\frac{l^{2} M}{2}\left[1 \pm \sqrt{1-\left(\frac{J}{M l}\right)^{2}}\right]$.

Another noncommutative BTZ solution has been worked out in the polar coordinates with a different commutation relation, more details could be found in Ref. [46].

The above metric shows an interesting feature which differs from the commutative case: The apparent and Killing horizons do not coincide except for the non-rotating case (see [32]). For simplicity, we just investigate non-rotating noncommutative BTZ black hole by setting the angular momentum $J=0$, we also set the radius of the anti-de Sitter space to be unity, i.e. $l=1$, thus the metric of noncommutative BTZ black hole is given as

$d s^{2}=-f(r) d t^{2}+g(r)^{-1} d r^{2}+K d \phi^{2}$,

where

$$
\begin{aligned}
& f(r)=-M+r^{2}-\frac{\theta B}{2}, \\
& g(r)=-M+r^{2}-\frac{\theta B}{2}\left(2-\frac{M}{r^{2}}\right),
\end{aligned}
$$

$$
K=r^{2}+\frac{\theta B}{2} .
$$

We can obtain the radius of event horizon from

$g\left(r_{h}\right)=0$.

The solutions of the above equation are

$$
\begin{aligned}
\left(r_{h}^{2}\right)_{\frac{1}{2}} & =\frac{1}{2}\left(M+\theta B \pm \sqrt{M^{2}+\theta^{2} B^{2}}\right) \\
& \cong \frac{1}{2}\left(M+\theta B \pm M+\mathcal{O}\left(\theta^{2}\right)\right) .
\end{aligned}
$$

The analytical expressions of the two distinct solutions for the horizon are

$$
\begin{aligned}
& \left(r_{h}^{2}\right)_{1}=M+\frac{\theta B}{2}+\mathcal{O}\left(\theta^{2}\right), \\
& \left(r_{h}^{2}\right)_{2}=\frac{\theta B}{2}+\frac{1}{2} \mathcal{O}\left(\theta^{2}\right) .
\end{aligned}
$$

Since there is no obviously physical meaning without black hole mass term in $\left(r_{h}^{2}\right)_{2}$, we will not consider this case. Thus, $g(r)$ can be rewritten as

$$
g(r)=-M+r^{2}-\frac{\theta B}{2}\left[2-\frac{r_{h}^{2}-\frac{\theta B}{2}-\mathcal{O}\left(\theta^{2}\right)}{r^{2}}\right] .
$$

Ignoring the higher order of the noncommutative parameter $\theta$, we have

$g(r)=-M+r^{2}-\frac{\theta B}{2}\left[2-\frac{r_{h}^{2}}{r^{2}}\right]$,

obviously, $g(r)_{r \simeq r_{h}} \simeq f(r)_{r \simeq r_{h}}$ in the region $\left(r \simeq r_{h}\right)$. But when $r>>r_{h}, g(r)_{r>>r_{h}}=-M+r^{2}-\theta B=f(r)-\frac{\theta B}{2}$, the second term $\frac{\theta B}{2}$ in $g(r)$ would be considered as a correction term in the asymptotic region $\left(r>>r_{h}\right)$, i.e. $g(r)_{r>>r_{h}} \simeq f(r)_{r>>r_{h}}$.

\section{Scalar QNM in the noncommutative BTZ background}

The quasinormal modes of the noncommutative massless scalar field have been well studied in Ref. [24] in a massive non-rotating BTZ black hole background up to the first order in the deformation parameter. The equations of motion governing these quasinormal modes are identical to the equations of motion of a commutative massive scalar field in a fictitious massive spinning BTZ black hole background. These results hint at a duality between the commutative and noncommutative systems in the background of the BTZ black hole. They also suggested that the noncommutativity of the scalar field is possibly raised by the back-reaction, additional mass and angular momentum of the system with the fictitious black hole. In order to check this interesting conjecture, we will firstly explore this noncommutative BTZ black hole in nonminimally coupling scalar field, which can be described by 
Klein-Gorden equation (see [47])

$\frac{1}{\sqrt{-g}} \partial_{\mu}\left(\sqrt{-g} g^{\mu \nu} \partial_{\nu}\right) \Phi=\xi R_{3}$,

where $\xi$ is the coupling between the scalar and the gravitational fields, additionally, the nonminimal coupling is taken to be positive. The Ricci scalar of the BTZ background is $R_{3}=-\frac{6}{l^{2}}$.

Using the ansatz

$\Phi(t, r, \phi)=e^{-i \omega t} R(r) e^{i m \phi}$.

We can obtain ordinary differential equations which consist of the radial and angular equations. The radial equation is

$$
\begin{aligned}
& R^{\prime \prime}+\left(\frac{f^{\prime}(r)}{2 f(r)}+\frac{g^{\prime}(r)}{2 g(r)}+\frac{K^{\prime}}{2 K}\right) R^{\prime}+\left(\frac{\omega^{2}}{f(r) g(r)}\right. \\
& \left.-\frac{m^{2}}{g(r) K}-\frac{\xi R_{3}}{g(r)}\right) R=0 .
\end{aligned}
$$

In order to solve the Eq. (22), we define a new dimensionless parameter $z=1-\frac{r_{h}^{2}}{r^{2}}$, which ranges from $0\left(r \simeq r_{h}\right)$ to $1\left(r>>r_{h}\right)$, thus the Eq. (22) becomes a hypergeometric equation

$z(1-z) \frac{d^{2} R}{d z^{2}}+(1-z) \frac{d R}{d z}+\left(\frac{A}{z}+\frac{B}{-1+z}-C\right) R=0$,

where

$A=\frac{\omega^{2}}{4 r_{h}^{2}}$,

$B=-\frac{6 \xi}{4}$,

$C=\frac{m^{2}}{4 K_{h}}$

and $K_{h}=r_{h}^{2}+\frac{\theta B}{2} \simeq r_{h}^{2}$. In order to analytically solve the hypergeometric Eq. (23), we assume that the radial part $R(z)$ takes the form as

$R(z)=z^{\alpha_{s}}(1-z)^{\beta_{s}} F\left(a_{s}, b_{s}, c_{s}, z\right)$.

Inserting $R(z)$ into the Eq. (23)

$$
\begin{aligned}
& z(1-z) \frac{d^{2} F}{d z^{2}}+\left[1+2 \alpha_{s}-\left(1+2 \alpha_{s}+2 \beta_{s}\right) z\right] \frac{d F}{d z} \\
& +\left(\frac{\bar{A}}{z}+\frac{\bar{B}}{-1+z}-\bar{C}\right) F=0,
\end{aligned}
$$

where

$$
\begin{aligned}
\bar{A} & =\alpha_{s}^{2}+A, \\
\bar{B} & =-\beta_{s}\left(\beta_{s}-1\right)+B, \\
\bar{C} & =\left(\alpha_{s}+\beta_{s}\right)^{2}+C .
\end{aligned}
$$

Setting that $\bar{A}=\bar{B}=0$, we obtain the parameters $\alpha_{s}$ and $\beta_{s}$

$\alpha_{s}= \pm \frac{i \omega}{2 r_{h}}$

$\beta_{s}=\frac{(1 \pm \sqrt{1-6 \xi})}{2}$.

For generality, we choose $\alpha_{s}=-\frac{i \omega}{2 r_{h}}$ and $\beta_{s}=\frac{(1-\sqrt{1-6 \xi})}{2}$.

The general form of a hypergeometric equation is

$$
\begin{aligned}
& z(1-z) \frac{d^{2} F}{d z^{2}}+\left[c_{s}-\left(1+a_{s}+b_{s}\right) z\right] \frac{d F}{d z} \\
& \quad-a_{s} b_{s} F=0
\end{aligned}
$$

thus the exact expressions of $a_{s}, b_{s}, c_{s}$ can be obtained by combining Eqs. (28) and (34)

$$
\begin{aligned}
& c_{s}=1+2 \alpha_{s}, \\
& a_{s}=\alpha_{s}+\beta_{s}+\frac{i m}{2 r_{h}}, \\
& b_{s}=\alpha_{s}+\beta_{s}-\frac{i m}{2 r_{h}} .
\end{aligned}
$$

In the near-horizon region, two linearly independent solutions of Eq. (34) have been studied in Ref. [48], which are $F\left(a_{s}, b_{s}, c_{s}, z\right)$ and $z^{1-c_{s}} F\left(a_{s}-c_{s}+1, b_{s}-c_{s}+1,2-c_{s}, z\right)$. The QNM are defined as solutions of purely ingoing wave near the horizon and outgoing wave vanishing at infinity [7]. The solution near the horizon can be described as

$R(z)=z^{\alpha_{s}}(1-z)^{\beta_{s}} F\left(a_{s}, b_{s}, c_{s}, z\right)$.

In the asymptotic region, we use the linear transformation formula $[2,48]$ to implement the vanishing boundary condition (Dirichlet boundary condition)

$$
\begin{aligned}
R(z)= & z^{\alpha_{s}}(1-z)^{1-\beta_{s}}(1-z)^{a_{s}+b_{s}-c_{s}} \frac{\Gamma\left(c_{s}\right) \Gamma\left(c_{s}-a_{s}-b_{s}\right)}{\Gamma\left(a_{s}\right) \Gamma\left(b_{s}\right)} \\
& \times F\left(c_{s}-a_{s}, c_{s}-b_{s}, c_{s}-a_{s}-b_{s}+1 ; 1-z\right) \\
& +z^{\alpha_{s}}(1-z)^{1-\beta_{s}} \frac{\Gamma\left(c_{s}\right) \Gamma\left(c_{s}-a_{s}-b_{s}\right)}{\Gamma\left(c_{s}-a_{s}\right) \Gamma\left(c_{s}-b_{s}\right)} \\
& \times F\left(a_{s}, b_{s}, a_{s}+b_{s}-c_{s}+1 ; 1-z\right) .
\end{aligned}
$$

Clearly, when $z=1$, the first term of above equation vanishes, but the vanishing of the second term requires meeting the restriction, $c_{s}-a_{s}=-n$ or $c_{s}-b_{s}=-n$. Thus, the left and right QNM frequencies take the form as follow

$$
\begin{aligned}
& \omega_{L}=m-2 i r_{h}\left(n+\frac{1}{2}+\frac{\sqrt{1-6 \xi}}{2}\right), \\
& \omega_{R}=-m-2 i r_{h}\left(n+\frac{1}{2}+\frac{\sqrt{1-6 \xi}}{2}\right) .
\end{aligned}
$$

Particularly, if we set the noncommutative parameter $\theta=0$ and the coupling constant $\xi=0$, our results completely reduce to the results in Refs. [2,24]. From our results, we find that the coupling constant $\xi$ will shift the QNM frequencies. For straightly seeing how the QNM frequencies $\omega$ depend on 
the noncommutative parameter $\theta$, we expand $r_{h}$ in terms of the noncommutative parameter $\theta$

$r_{h}=\sqrt{M}+\frac{\theta B}{4 \sqrt{M}}+\mathcal{O}\left(\theta^{2}\right)$

therefore, the QNM frequencies change into the form

$$
\begin{aligned}
\omega_{L}= & m-2 i \sqrt{M}\left(n+\frac{1}{2}+\frac{\sqrt{1-6 \xi}}{2}\right) \\
& -2 i\left(\frac{\theta B}{4 \sqrt{M}}+\mathcal{O}\left(\theta^{2}\right)\right)\left(n+\frac{1}{2}+\frac{\sqrt{1-6 \xi}}{2}\right), \\
\omega_{R}= & -m-2 i \sqrt{M}\left(n+\frac{1}{2}+\frac{\sqrt{1-6 \xi}}{2}\right) \\
& -2 i\left(\frac{\theta B}{4 \sqrt{M}}+\mathcal{O}\left(\theta^{2}\right)\right)\left(n+\frac{1}{2}+\frac{\sqrt{1-6 \xi}}{2}\right) .
\end{aligned}
$$

Equations $(43,44)$ are the exact expressions of the QNM frequencies for the noncommutative BTZ black hole with nonminimally coupling scalar field. Moreover, the noncommutativity of the BTZ black hole is also possibly raised by the magnetic field compared to the above conjecture.

\section{Fermionic QNM in the noncommutative BTZ background}

The fermionic QNM in the BTZ background have been numerically analysed in Refs. [8,25]. In this paper, we will devote to the QNM of the massless Dirac field in the noncommutative BTZ background. The Dirac equation in a general background space-time [49]

$\left[\gamma^{a} e_{a}^{\mu}\left(\partial_{\mu}+\Gamma_{\mu}\right)\right] \Psi=0$,

where $e_{a}{ }^{\mu}$ is the inverse of the tetrad $e_{\mu}{ }^{a}$ defined by metric

$g_{\mu \nu}=\eta_{a b} e_{\mu}{ }^{a} e_{\nu}^{b}$,

where the tetrad $e_{\mu}{ }^{a}=\operatorname{diag}\left(\sqrt{f}, \frac{1}{\sqrt{g}}, \sqrt{K}\right)$ and the Minkowski metric $\eta_{a b}=\operatorname{diag}(-1,1,1)$. The Dirac matrices $\gamma^{a}$ are (see [25])

$\gamma^{0}=i \sigma_{2}=\left(\begin{array}{cc}0 & 1 \\ -1 & 0\end{array}\right), \quad \gamma^{1}=\sigma_{1}=\left(\begin{array}{ll}0 & 1 \\ 1 & 0\end{array}\right)$,

$\gamma^{2}=\sigma_{3}=\left(\begin{array}{cc}1 & 0 \\ 0 & -1\end{array}\right)$,

where $\sigma_{i}(i=1,2,3)$ are the usual Pauli metrics. The spin connection $\Gamma_{\mu}$ reads

$\Gamma_{\mu}=\frac{1}{8}\left[\gamma^{a}, \gamma^{b}\right] e_{a}^{v} e_{b v ; \mu}$,

where $e_{b v ; \mu}=\partial_{\mu} e_{b v}-\Gamma_{\mu \nu}^{\alpha} e_{b \alpha}, \Gamma_{\mu \nu}^{\alpha}$ are the Christoffel symbols. In the noncommutative BTZ background, its non- vanishing Christoffel symbols are

$\Gamma_{01}^{0}=\Gamma_{10}^{0}=\frac{f^{\prime}}{2 f}, \quad \Gamma_{00}^{1}=\frac{g f^{\prime}}{2}, \quad \Gamma_{11}^{1}=-\frac{g^{\prime}}{2 g}$,

$\Gamma_{22}^{1}=-\frac{g K^{\prime}}{2}, \quad \Gamma_{21}^{2}=\Gamma_{12}^{2}=\frac{K^{\prime}}{2 K}$.

Inserting these Christoffel symbols into Eq. (47)

$$
\begin{aligned}
& \Gamma_{0}=-\frac{1}{4} \gamma^{0} \gamma^{1} \frac{\sqrt{g} f^{\prime}}{\sqrt{f}}, \\
& \Gamma_{1}=0 \\
& \Gamma_{2}=-\frac{1}{4} \gamma^{1} \gamma^{2} \frac{\sqrt{g} K^{\prime}}{\sqrt{K}} .
\end{aligned}
$$

The Dirac equation(45) becomes

$$
\left[\frac{\gamma^{0}}{\sqrt{f}} \partial_{t}+\gamma^{1} \sqrt{g}\left(\partial_{r}+\frac{f^{\prime}}{4 f}+\frac{K^{\prime}}{4 K}\right)+\frac{\gamma^{2}}{\sqrt{K}} \partial_{\phi}\right] \Psi=0 .
$$

Using the ansatz

$$
\Phi=e^{-i \omega t+i m \phi}\left(\begin{array}{l}
P+Q \\
P-Q
\end{array}\right)
$$

the Eq. (52) reduces to the form

$$
\begin{aligned}
& -\frac{i \omega}{\sqrt{f}}\left(\begin{array}{c}
P-Q \\
-P-Q
\end{array}\right)+\frac{i m}{\sqrt{K}}\left(\begin{array}{c}
P+Q \\
-P+Q
\end{array}\right) \\
& +\sqrt{g} \partial_{r}\left(\begin{array}{c}
P-Q \\
P+Q
\end{array}\right)+\sqrt{g}\left(\frac{f^{\prime}}{4 f}+\frac{K^{\prime}}{4 K}\right)\left(\begin{array}{l}
P-Q \\
P+Q
\end{array}\right)=0 .
\end{aligned}
$$

Setting

$$
\begin{aligned}
& P=\sqrt{\frac{\sqrt{\sqrt{f}+\sqrt{K}}}{\sqrt{f K}}} P^{\prime}, \\
& Q=\sqrt{\frac{\sqrt{\sqrt{f}-\sqrt{K}}}{\sqrt{f K}}} Q^{\prime},
\end{aligned}
$$

The Eq. (54) changes into

$$
\left\{\begin{array}{l}
\sqrt{g} \partial_{r}\left(\sqrt{\frac{\sqrt{f}+\sqrt{K}}{\sqrt{f K}}} P^{\prime}\right)+\frac{i \omega}{\sqrt{f}} \sqrt{\frac{\sqrt{f}-\sqrt{K}}{\sqrt{f K}}} Q^{\prime}+\frac{i m}{\sqrt{K}} \sqrt{\frac{\sqrt{f}-\sqrt{K}}{\sqrt{f K}}} Q^{\prime} \\
+\sqrt{g}\left(\frac{f^{\prime}}{4 f}+\frac{K^{\prime}}{4 K}\right) \sqrt{\frac{\sqrt{f}+\sqrt{K}}{\sqrt{f K}}} P^{\prime}=0 \\
\sqrt{g} \partial_{r}\left(\sqrt{\frac{\sqrt{f}-\sqrt{K}}{\sqrt{f K}}} Q^{\prime}\right)+\frac{i \omega}{\sqrt{f}} \sqrt{\frac{\sqrt{f}+\sqrt{K}}{\sqrt{f K}}} P^{\prime}-\frac{i m}{\sqrt{K}} \sqrt{\frac{\sqrt{f}+\sqrt{K}}{\sqrt{f K}}} P^{\prime} \\
+\sqrt{g}\left(\frac{f^{\prime}}{4 f}+\frac{K^{\prime}}{4 K}\right) \sqrt{\frac{\sqrt{f}-\sqrt{K}}{\sqrt{f K}}} Q^{\prime}=0,
\end{array}\right.
$$


therefore

$$
\left\{\begin{array}{l}
\sqrt{g} \sqrt{\frac{\sqrt{f}+\sqrt{K}}{\sqrt{f K}}} \partial_{r} P^{\prime}+\frac{i \omega}{\sqrt{f}} \sqrt{\frac{\sqrt{f}-\sqrt{K}}{\sqrt{f K}}} Q^{\prime}+\frac{i m}{\sqrt{K}} \sqrt{\frac{\sqrt{f}-\sqrt{K}}{\sqrt{f K}}} Q^{\prime} \\
+\frac{\sqrt{g}}{\sqrt[4]{\frac{\sqrt{f}+\sqrt{K}}{\sqrt{f K}}}}\left(\frac{K^{\prime}}{K \sqrt{f}}+\frac{f^{\prime}}{f \sqrt{K}}\right) P^{\prime}=0, \\
\sqrt{g} \sqrt{\frac{\sqrt{f}-\sqrt{K}}{\sqrt{f K}}} \partial_{r} Q^{\prime}+\frac{i \omega}{\sqrt{f}} \sqrt{\frac{\sqrt{f}+\sqrt{K}}{\sqrt{f K}}} P^{\prime}-\frac{i m}{\sqrt{K}} \sqrt{\frac{\sqrt{f}+\sqrt{K}}{\sqrt{f K}}} P^{\prime} \\
-\frac{\sqrt{g}}{\sqrt[4]{\frac{\sqrt{f}-\sqrt{K}}{\sqrt{f K}}}}\left(\frac{K^{\prime}}{K \sqrt{f}}+\frac{f^{\prime}}{f \sqrt{K}}\right) Q^{\prime}=0 .
\end{array}\right.
$$

By assuming $g(r) \simeq f(r)$, we rewrite the Eq. (57) with dimensionless parameter $z$

$\left\{\begin{array}{l}2 \sqrt{z}(1-z) \frac{d P^{\prime}}{d z}+X\left(\frac{1}{\sqrt{z}}-1\right) Q^{\prime}+Y(1-\sqrt{z}) Q^{\prime}+\frac{1}{2} P^{\prime}=0, \\ 2 \sqrt{z}(1-z) \frac{d Q^{\prime}}{d z}+X\left(\frac{1}{\sqrt{z}}-1\right) P^{\prime}-Y(1-\sqrt{z}) P^{\prime}-\frac{1}{2} Q^{\prime}=0,\end{array}\right.$

where, $X=\frac{i \omega}{r_{h}}, Y=\frac{i m}{r_{h}}$. By making a further transformation

$\left\{\begin{array}{l}P^{\prime}=\psi_{1}+\psi_{2} \\ Q^{\prime}=\psi_{1}-\psi_{2}\end{array}\right.$

we can obtain

$$
\left\{\begin{array}{l}
2 \sqrt{z}(1-z) \frac{d \psi_{1}}{d z}+\left(\frac{X}{\sqrt{z}}-Y \sqrt{z}\right) \psi_{1}+\left(X-Y+\frac{1}{2}\right) \psi_{2}=0, \\
2 \sqrt{z}(1-z) \frac{d \psi_{2}}{d z}+\left(-\frac{X}{\sqrt{z}}+Y \sqrt{z}\right) \psi_{2}+\left(-X+Y+\frac{1}{2}\right) \psi_{1}=0 .
\end{array}\right.
$$

Combining these two equations, we arrive at a hypergeometric equation

$$
\begin{aligned}
& z(1-z) \frac{d^{2} \psi_{1}}{d z^{2}}+\frac{1-3 z}{2} \frac{d \psi_{1}}{d z}+\frac{1}{4}\left[\frac{-X^{2}-X}{z}\right. \\
& \left.+\left(Y^{2}-Y\right)-\frac{1}{4(1-z)}\right] \psi_{1}=0,
\end{aligned}
$$

the solution to this hypergeometric equation is

$$
\begin{aligned}
\psi_{1}= & z^{\alpha_{f}}(1-z)^{\beta_{f}}\left(F\left(a_{f}, b_{f}, c_{f} ; z\right)+z^{1-c_{f}} F\left(a_{f}-c_{f}\right.\right. \\
& \left.\left.+1, b_{f}-c_{f}+1,2-c_{f} ; z\right)\right),
\end{aligned}
$$

where

$$
\begin{aligned}
& \alpha_{f}=-\frac{X}{2}, \\
& \beta_{f}=-\frac{1}{4}, \\
& a_{f}=\alpha_{f}+\beta_{f}+\frac{1}{2}-\frac{Y}{2}, \\
& b_{f}=\alpha_{f}+\beta_{f}+\frac{Y}{2},
\end{aligned}
$$

$c_{f}=\frac{1}{2}+2 \alpha_{f}$.

With the similar method we can get $\psi_{2}$, so $\psi_{1}$ and $\psi_{2}$ at the horizon are given as

$$
\begin{aligned}
& \psi_{1}=z^{\alpha_{f}}(1-z)^{\beta_{f}} F\left(a_{f}, b_{f}, c_{f}, z\right), \\
& \psi_{2}=\left(\frac{a_{f}-c_{f}}{c_{f}}\right) z^{\alpha_{f}+\frac{1}{2}}(1-z)^{\beta_{f}} F\left(a_{f}, b_{f}+1, c_{f}+1, z\right) .
\end{aligned}
$$

In analogy with scalar QNM, the asymptotic form $(z \rightarrow 1)$ of $\psi_{1}$ and $\psi_{2}$ can be determined exactly by implementing the boundary condition, i.e. Dirichlet condition. Therefore, the vanishing of the fermionic flux at the asymptotic regime imposes the requirements $c_{f}-a_{f}=-n$ or $c_{f}-b_{f}=-n$

$$
\begin{aligned}
& \omega_{L}=m-2 i r_{h}\left(n+\frac{1}{4}\right), \\
& \omega_{R}=-m-2 i r_{h}\left(n+\frac{3}{4}\right) .
\end{aligned}
$$

The contribution of the noncommutative parameter consists in $r_{h}$, we also can express the QNM frequencies with the noncommutative parameter

$$
\begin{aligned}
\omega_{L}= & m-2 i \sqrt{M}\left(n+\frac{1}{4}\right) \\
& -2 i\left(\frac{\theta B}{4 \sqrt{M}}+\mathcal{O}\left(\theta^{2}\right)\right)\left(n+\frac{1}{4}\right), \\
\omega_{R}= & -m-2 i \sqrt{M}\left(n+\frac{3}{4}\right)-2 i\left(\frac{\theta B}{4 \sqrt{M}}\right. \\
& \left.+\mathcal{O}\left(\theta^{2}\right)\right)\left(n+\frac{3}{4}\right) .
\end{aligned}
$$

These two equations are the exact expressions of the QNM frequencies for the noncommutative BTZ black hole in massless Dirac field.

\section{Quantitative agreement in the noncommutative BTZ background}

In Ref. [7], Horowitz et al. have found that a correspondence exists between QNM and the decay of perturbations in the dual conformal field theory $(C F T)$. They have simulated the QNM for high-dimension $A d S$-Schwarzschild black holes. Further numerical simulations of quasi-normal modes in asymptotically $A d S$ space-times have been presented in Refs. [50-55]. Birmingham et al. [8] have showed that there is a precise quantitative agreement between QNM frequencies and the location of the poles of the retarded correlation function describing the linear response on the conformal field theory in the BTZ background. The dual conformal field theory on the boundary is $1+1$ dimensional, the conformal symmetry being generated by two copies of the Virasoro 
algebra acting separately on left- and right-moving sectors. Consequently, the conformal field theory splits into two independent sectors at thermal equilibrium with temperatures

$T_{L}=\left(r_{+}-r_{-}\right) / 2 \pi$,

$T_{R}=\left(r_{+}+r_{-}\right) / 2 \pi$.

According to the $A d S_{3} / C F T_{2}$ correspondence, the field of spin $s$ propagating in $A d S_{3}$ corresponds to conformal weights $\left(h_{L}, h_{R}\right)$ in the dual conformal field theory [6]

$h_{R}+h_{L}=\Delta$,

$h_{R}-h_{L}= \pm s$,

where $\Delta$ is determined by the $m$ of the field. In particular, $\Delta=1+\sqrt{1+m^{2}}$ is for scalar field, $\Delta=1+|m|$ is for fermionic field.

The decay of the perturbation on the $C F T$ which coincides precisely with the QNM of the BTZ black hole was presented (more details in Ref. [8])

$\omega_{L}=k-4 \pi i T_{L}\left(n+h_{L}\right)$,

$\omega_{R}=-k-4 \pi i T_{R}\left(n+h_{R}\right)$,

By adopting the above methods for the noncommutative BTZ black hole case, we have the thermal equilibrium with temperatures

$T_{L}^{\theta}=T_{R}^{\theta}=r_{h} / 2 \pi=\left(\sqrt{M}+\frac{\theta B}{4 \sqrt{M}}+\mathcal{O}\left(\theta^{2}\right)\right) / 2 \pi$,

this formulation suggests that the noncommutativity leads to the fluctuation of the thermal equilibrium. According to the exact expressions of scalar and fermionic QNM for the noncommutative BTZ black hole, we have obtained

(I) The scalar case:

$$
\begin{aligned}
h_{L}^{s} & =h_{R}^{s}=\frac{1}{2}+\frac{\sqrt{1-6 \xi}}{2}, \\
h_{R}^{s}+h_{L}^{s} & =1+\sqrt{1-6 \xi}, \\
h_{R}^{s}-h_{L}^{s} & =0,
\end{aligned}
$$

(II) The fermionic case:

$$
\begin{aligned}
& h_{L}^{f}=\frac{1}{4}, \quad h_{R}^{f}=\frac{3}{4}, \\
& h_{R}^{f}+h_{L}^{f}=1, \\
& h_{R}^{f}-h_{L}^{f}=\frac{1}{2} .
\end{aligned}
$$

Particularly, our results agrees with Ref. [8] if we treat the coupling constant as a new negative massive parameter square (i.e. $6 \xi=-m^{2}$ ) for the scalar field. Our results suggest that the quantitative agreement also exists between the QNM of the noncommutative BTZ black hole and the poles of the retarded correlation function of the corresponding perturbations of the dual conformal field theory. We also have found that the noncommutativity of BTZ black hole does not affect conformal weights $\left(h_{L}, h_{R}\right)$, but influences the thermal equilibrium.

\section{Quantization of area/entropy}

In quantum gravity theory, the black hole area should be represented by a quantum operator with a discrete spectrum of eigenvalues. The horizon area of black hole is supposed to be an adiabatic invariant which leads to an equidistant area spectrum [26]. In Ref. [56], the authors have pointed out that the ratio $\frac{E}{\Delta \omega_{E}}$ is an adiabatic invariant, where $E$ and $\Delta \omega_{E}$ are the energy and vibrational frequency. It is widely believed that the oscillating black hole radiates or absorbs elementary quanta of energy/mass when it undergoes a transition between two quantum states in its QNM spectrum. However, its QNM frequencies are complex quantities which lead to a serious problem, i.e. which part of them carries the genuine information about the energy. In our paper, we replace the energy $E$ with mass $M$ and obtain vibrational frequency between the two adjacent states of the black hole by following the same methods in Ref. [57]. The radius of event horizon has been given in Eq. (16) and the area of this black hole has the form

$A=2 \pi \sqrt{M+\frac{\theta B}{2}+\mathcal{O}\left(\theta^{2}\right)}$.

The adiabatic invariant $I$ for this noncommutative BTZ black hole is

$I=\int \frac{d M}{\Delta \omega}$.

In the case of $n \rightarrow \infty$, the vibrational frequency can be obtained from the QNM frequencies we have presented

$\Delta \omega=\left(\left|\omega_{I}\right|\right)_{n}-\left(\left|\omega_{I}\right|\right)_{n-1}=2 \sqrt{M+\frac{\theta B}{2}+\mathcal{O}\left(\theta^{2}\right)}$.

Substituting $\Delta \omega$ into Eq. (88), the exact expression of the adiabatic invariant takes the form as follow

$I=\sqrt{M+\frac{\theta B}{2}+\mathcal{O}\left(\theta^{2}\right)}$.

By Taylor expansion of the adiabatic invariant $I$ around $\theta=$ 0 , we obtain

$I=\sqrt{M}+\frac{\theta B}{4 \sqrt{M}}+\mathcal{O}\left(\theta^{2}\right)$.

In Ref. [57] the relevant quantities of the non-rotating BTZ black hole are given as

$$
\begin{aligned}
I & =\sqrt{M}, \\
A_{n} & =2 \pi n \hbar \sqrt{\frac{1}{\Lambda},}
\end{aligned}
$$


$S_{n}=\frac{1}{2} \pi n \hbar \sqrt{\frac{1}{\Lambda}}$,

where, $\Lambda=\frac{1}{l^{2}}$. The spacing of the area or entropy are given as

$\Delta A=2 \pi \hbar \sqrt{\frac{1}{\Lambda}}$.

From the above equation, we find that the area spectrum is equidistant in high damped modes. Comparing Eqs. (91) with (92), we note that the Eq. (91) has a noncommutative term which is more complex than Eq. (92). In Ref. [24], the adiabatic invariant contains the deformation parameter term. In order to make the noncommutative part more intuitive and convenient to investigate, we can divide the adiabatic invariant into commutative part and noncommutative part in the Bohr-Sommerfeld quantization condition

$I_{\text {sum }}=I_{\text {non }}+I_{\text {com }}$.

Using the Bohr-Sommerfeld quantization, we may get

$I_{\text {sum }}=I_{\text {non }}+I_{\text {com }}=n_{\text {non }} \hbar+n_{\text {com }} \hbar$.

From Eqs. (91) and (97), we obtain

$$
\begin{aligned}
\sqrt{M} & =n_{c o m} \hbar, \\
\frac{\theta B}{4 \sqrt{M}}+\mathcal{O}\left(\theta^{2}\right) & =n_{\text {non }} \hbar .
\end{aligned}
$$

Therefore, the exact expression of the area spectrum of noncommutative BTZ black hole takes the form

$A_{n}=2 \pi\left(n_{c o m}+n_{\text {non }}\right) \hbar$.

The entropy spectrum of this black hole is

$S_{n}=\frac{1}{2} \pi\left(n_{\text {com }}+n_{\text {non }}\right) \hbar$.

The distant of two adjacent states for area/entropy spectrum is

$$
\begin{aligned}
\Delta A_{n}= & A_{n}-A_{n-1}=2 \pi\left(n_{\text {com }}+n_{n o n}\right) \hbar \\
& -2 \pi\left(n_{\text {com }}-1+n_{\text {non }}^{\prime}\right) \hbar=2 \pi\left(1+n_{\text {non }}^{\prime \prime}\right) \hbar . \\
\Delta S_{n}= & S_{n}-S_{n-1}=\frac{1}{2} \pi\left(n_{\text {com }}+n_{n o n}\right) \hbar \\
& -\frac{1}{2} \pi\left(n_{\text {com }}-1+n_{\text {non }}^{\prime}\right) \hbar=\frac{1}{2} \pi\left(1+n_{\text {non }}^{\prime \prime}\right) \hbar .
\end{aligned}
$$

It is obvious that both the area spectrum and entropy spectrum are not equidistant because of the existence of noncommutative term. Particularly, it is the noncommutativity of BTZ black hole that brings in this additional richness and complexity into the pattern of the area/entropy spectrum, leading to its nonuniform. The area/entropy spectrum of these two systems can be described by the corresponding discrete spectra with non-equidistant values. Moreover, we can find that the coupling constant $\xi$ does not exist in the area/entropy spectrum, it has been dropped when we calculate vibrational frequency.
Therefore, the coupling constant will not contribute to the nonuniform of area/entropy. That's to say, the BTZ black holes with nonminimally coupling scalar field have the same area/entropy spectrum with the BTZ black hole in minimally coupling scalar field.

\section{Conclusion}

In summary, we have investigated the quasinormal modes and area/entropy spectrum for the noncommutative BTZ black hole. The analytical expressions for the scalar and feimionic QNM have been given in noncommutative BTZ background. Our results of QNM frequencies could reduce to the formula in Refs. $[8,24,25]$ when the noncommutative parameters and the coupling constant disappear. We have found that the coupling constant $\xi$ shifts the QNM frequencies but does not affect the structure of the area/entropy spectrum. We also have found that the noncommutativity does not affect conformal weights $\left(h_{L}, h_{R}\right)$, but influences the thermal equilibrium, which suggests that the quantitative agreement also exists between the QNM of the noncommutative BTZ black hole and the poles of the retarded correlation function of the corresponding perturbations of the dual conformal field theory. According to Bohr-Sommerfeld quantization, we have presented the intuitive expressions of the area/entropy spectrum of the noncommutative BTZ black hole with the nonminimally coupling scalar field. Clearly, the area/entropy spectrum are not equidistant because of the existence of noncommutativity. Particularly, it is noncommutativity of this black hole that brings in this additional richness and complexity into the pattern of the area/entropy spectrum, leading to its nonuniform.

Acknowledgements This work is supported by the National Natural Science Foundation of China under Grant nos. U1731107.

Open Access This article is distributed under the terms of the Creative Commons Attribution 4.0 International License (http://creativecomm ons.org/licenses/by/4.0/), which permits unrestricted use, distribution, and reproduction in any medium, provided you give appropriate credit to the original author(s) and the source, provide a link to the Creative Commons license, and indicate if changes were made.

Funded by $\mathrm{SCOAP}^{3}$.

\section{References}

1. E. Berti, V. Cardoso, A.O. Starinets, Class. Quantum Gravity 26, 163001 (2009)

2. D. Birmingham, Phys. Rev. D 64, 064024 (2001)

3. T. Regge, J.A. Wheeler, Phys. Rev. 108, 1063 (1957)

4. C.V. Vishveshwara, Nature 227, 936 (1970)

5. R.A. Konoplya, A. Zhidenko, Rev. Mod. Phys. 83, 793 (2011)

6. O. Aharony, S.S. Gubser, J.M. Maldacena, H. Ooguri, Y. Oz, Phys. Rep. 323, 183 (2000) 
7. G.T. Horowitz, V.E. Hubeny, Phys. Rev. D 62, 024027 (2000)

8. D. Birmingham, I. Sachs, S.N. Solodukhin, Phys. Rev. Lett. 88, 151301 (2002)

9. D. Birmingham, I. Sachs, S.N. Solodukhin, Phys. Rev. D 67, 104026 (2003)

10. A. Connes, Noncommutative Geometry (Academic Press, San Diego, 1994)

11. C. Rovelli, L. Smolin, Phys. Rev. D 52, 10 (1995)

12. S. Doplicher, K. Fredenhagen, J.E. Roberts, Commun. Math. Phys. 172, 187 (1995)

13. B.P. Dolan, K.S. Gupta, A. Stern, Class. Quantum Gravity 24, 1647 (2007)

14. B.P. Dolan, K.S. Gupta, A. Stern, J. Phys. Conf. Ser. 174, 012023 (2009). (IOP Publishing)

15. T. Ohl, A. Schenkel, JHEP 0910, 052 (2009)

16. J. Lukierski, H. Ruegg, A. Nowicki, V.N. Tolstoi, Phys. Lett. B 264, 331 (1991)

17. J. Lukierski, H. Ruegg, Phys. Lett. B 329, 189 (1994)

18. J. Gomis, T. Mehen, Nucl. Phys. B 591, 265 (2000)

19. A.P. Balachandran, T.R. Govindarajan, G. Mangano, A. Pinzul, B.A. Qureshi, S. Vaidya, Phys. Rev. D 75, 045009 (2007)

20. A.P. Balachandran, P. Padmanabhan, A.R. de Queiroz, Phys. Rev. D 84, 065020 (2011)

21. T. Juric, T. Poulain, J.C. Wallet, JHEP 1605, 146 (2016)

22. A. Gere, T. Juric, J.C. Wallet, JHEP 1512, 045 (2015)

23. P. Aschieri, M. Dimitrijevic Ciric, R.J. Szabo, JHEP 1802, 036 (2018)

24. K.S. Gupta, E. Harikumar, T. Juri, JHEP 1509, 25 (2015)

25. K.S. Gupta, T. Juri, A. Samsarov, JHEP 1706, 107 (2017)

26. J.D. Bekenstein, Lett. Nuovo Cimento 11, 467 (1974)

27. C. Rovelli, L. Smolin, Nucl. Phys. B 442, 593 (1995)

28. C. Rovelli, L. Smolin, Nucl. Phys. B 456, 734 (1995)

29. A. Ashtekar, J. Lewandowski, Class. Quantum Gravity 14, 55 (1997)

30. S. Hod, Phys. Rev. Lett. 81, 4293 (1998)

31. M.R. Setare, Class. Quantum Gravity 21, 1453 (2004)

32. E. Chang-Young, D. Lee, Y. Lee, Class. Quantum Gravity 26, 185001 (2009)
33. N. Seiberg, E. Witten, JHEP 9909, 032 (1999)

34. A.H. Chamseddine, Phys. Lett. B 504, 33 (2001)

35. P. Aschieri, C. Blohmann, M. Dimitrijevi et al., Class. Quantum Gravity 22, 3511 (2005)

36. P. Aschieri, M. Dimitrijević, F. Meyer et al., Class. Quantum Gravity 23, 1883 (2006)

37. M. Chaichian, M. Oksanen, A. Tureanu et al., Phys. Rev. D 79, 044016 (2009)

38. M. Chaichian, P.P. Kulish, K. Nishijima, A. Tureanu et al., Phys. Lett. B 604, 98 (2004)

39. M. Chaichian, P. Presnajder, A. Tureanu, Phys. Rev. Lett. 94, $151602(2005)$

40. A. Achucarro, P.K. Townsend, Phys. Lett. B 180, 89 (1986)

41. E. Witten, Nucl. Phys. B 311, 46 (1988)

42. M. Banados, O. Chandia, N. Grandi et al., Phys. Rev. D 64, 084012 (2001)

43. S. Cacciatori, D. Klemm, L. Martucci et al., Phys. Lett. B 536, 101 (2002)

44. A. Connes, M.R. Douglas, A. Schwarz, JHEP 9802, 003 (1998)

45. M.R. Douglas, C. Hull, JHEP 9802, 008 (1998)

46. H.C. Kim, M.I. Park, C. Rim, JHEP 0810, 060 (2008)

47. G. Panotopoulos, Á. Rincón, Phys. Lett. B 772, 523 (2017)

48. M. Abramowitz, I.A. Stegun, Handbook of Mathematical Functions (Dover, New York, 1970)

49. D.R. Brill, J.A. Wheeler, Rev. Mod. Phys. 29, 465 (1957)

50. J.S. Chan, R.B. Mann, Phys. Rev. D 55, 7546 (1997)

51. B. Wang, C.Y. Lin, E. Abdalla, Phys. Lett. B 481, 79 (2000)

52. T.R. Govindarajan, V. Suneeta, Class. Quantum Gravity 18, 265 (2001)

53. V. Cardoso, J.P.S. Lemos, Phys. Rev. D 63, 124015 (2001)

54. V. Cardoso, J.P.S. Lemos, Phys. Rev. D 64, 084017 (2001)

55. B. Wang, E. Abdalla, R.B. Mann, Phys. Rev. D 65, 084006 (2002)

56. G. Kunstatter, Phys. Rev. Lett. 90, 161301 (2003)

57. Y.X. Liu, S.W. Wei, R. Li, JHEP 0903, 076 (2009) 